\title{
AP BASED PACKET LEVEL FEC MECHANISM USING ERED ALGORITHM FOR DATA TRANSMISSION
}

\author{
Archana S. Randive ${ }^{1} \&$ Veerbhadra S. Bale ${ }^{2}$
}

\begin{abstract}
Data transmission over the wireless network faces many challenges. The most critical challenge is related to packet loss. To overcome the problem of packet loss, Forward Error Correction is used by adding extra packets known as redundant packet or parity packet to the original information. In Conventional sender-based FEC the number of redundant packets added to the source packets remains constant irrespective of changes in the network condition. The recovery performance of such schemes is therefore somewhat unpredictable because they fail to capture the real-time network conditions and adjust the FEC redundancy rate accordingly. Thus, this paper proposes an FEC mechanism implemented at AP and the redundancy rate is tuned in accordance with both the wireless channel condition (as indicated by the number of packet retransmissions) and the network traffic load (as indicated by the AP queue length).
\end{abstract}

Keywords: Random Early Detection (RED), Forward Error Correction (FEC), Access Point (AP).

\section{INTRODUCTION}

Transmission of real-time video over the wireless network, usually, disturbed by the video packet loss that caused by interference, terrestrial obstructions and reflection of transmission signal [2-4]. Especially, high rate of packet loss significantly decreases the network throughput and video quality. To make sure that the video delivered at the receiver in good quality, two error correcting methods is used in general, such as Retransmission and FEC [5-8]. Retransmission is the most commonly used transport layer approach to recover packet errors and losses [9]. In retransmission-based error recovery, missing data packets are retransmitted during timeouts or explicit receiver's requests. A retransmission mechanism based on Automatic Repeat Request (ARQ) have been successfully used in the TCP protocol, FTP and Telnet as an error correction mechanism where guaranteed delivery is required. Retransmission mechanism is usually rejected as an error control method as it introduces retransmission (end-to-end) delays which could lead video packets to miss the play out time. By contrast, the principle of FEC is to add redundant packets together with the data packets at the sender so that original packets can be reconstructed at the receiver in the occurrence of packet loss without the need for retransmission of the last packet from the sender. Hence the FEC mechanism result in a lower retransmission latency and are therefore widely preferred for delivery of video streams over wireless networks [10].

\section{PROPOSED TECHNIQUE}

In the proposed approach, redundant FEC packets are generated dynamically at the AP in accordance with both the wireless channel condition and the traffic load of the current network. The channel condition is evaluated by monitoring the number of packet retransmissions. As the condition of the wireless channel deteriorates, a greater number of redundant FEC packets are generated. While, as the condition of the channel improves, the number of FEC packets is reduced. The network traffic load is evaluated by monitoring the queue length at the wireless AP. The queue length is short, if network load is low; queue length is long, if network load is high. When the network is lightly loaded, then more FEC packets can be generated. By the contrast, when the network is heavily loaded, then fewer FEC packets are generated to avoid unnecessary congestion of the network. By adopting this approach [1], proposed algorithm significantly improves the video quality without overloading the network with an excessive number of redundant packets.

Forward Error Correction

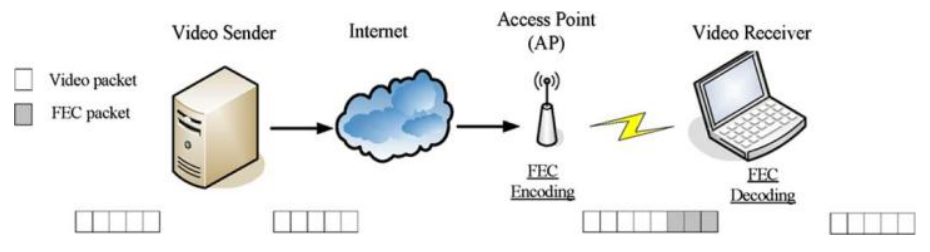

Figure 1. AP FEC mechanism

\footnotetext{
${ }^{1}$ PG Student, Department of Electronics Engineering, M.S. Bidve Engineering College, Latur (Maharashtra), India

${ }^{2}$ Associate Professor, Department of Electronics Engineering, M.S. Bidve Engineering College, Latur (Maharashtra), India
} 
Forward Error Correction (FEC) is one of the most widely utilized reliable, error correction/control techniques which have been successfully used in a number of application domains from space communication with supporting multimedia traffic with realtime constraints over wide-area high-speed networks [11].The basic principle of FEC is stated as [1], given a communication channel with known error probabilities, using error-correcting code to inject redundant packets called parity packets on the compressed source video packet streams at the sender so that the receivers may detect errors/loss and reconstruct the lost packet from $\mathrm{h}$ redundant packets without the need for retransmission of the lost packets from the sender. As shown in Fig. 1, the original block is encoded as ( $\mathrm{n}, \mathrm{k}$ ) packets, where $\mathrm{n}$ is the summation of source packets $\mathrm{k}$ and $\mathrm{h}$ redundant packets. Thus, provided that no more than $\mathrm{h}$ packets are lost in transmission, the source transmission packets can be successfully recovered at the receiver. As FEC schemes enable the recovery of lost source packets, the effective loss rate in the transmission network is lower than the actual loss rate. FEC can be applied at the packet-level and on the bit-level [12]. Normally packet-level FEC is applied to end-to-end communication, whereas bit-level FEC is used on a specific link [13]. When FEC is applied at the bitlevel some redundancy bits are added in each sent packet, hence the packet becomes larger. When FEC is applied to the packetlevel some redundancy packets are added as separate packets in each sent group of packets. Therefore, the sent group of packets (block) is composed of some redundancy packets and some data packets. Due to ARQ's inherent inadequacy in handling endto-end network latencies, the packet-level FEC scheme has become a suitable error control mechanism for real-time video transmission [14].

\section{QUEUE MANAGEMENT POLICY}

The management policy of queue adopted by the wireless AP will influence the network throughput, end-to-end delay and QoS. At the wireless AP, the RED algorithm [15] decides and selects the next packet to be transmitted. It also controls how to drop the packets when the queue length is full. The RED algorithm uses two queue length thresholds that trigger certain activity: Min Threshold and Max Threshold. When the packet arrives, RED compares the AvgLen with these two thresholds according to the rules shown in fig.2.

If the Avg length is lower than the lower threshold, no action is taken and if the Avg length is larger than the upper threshold, then the packet is always dropped.

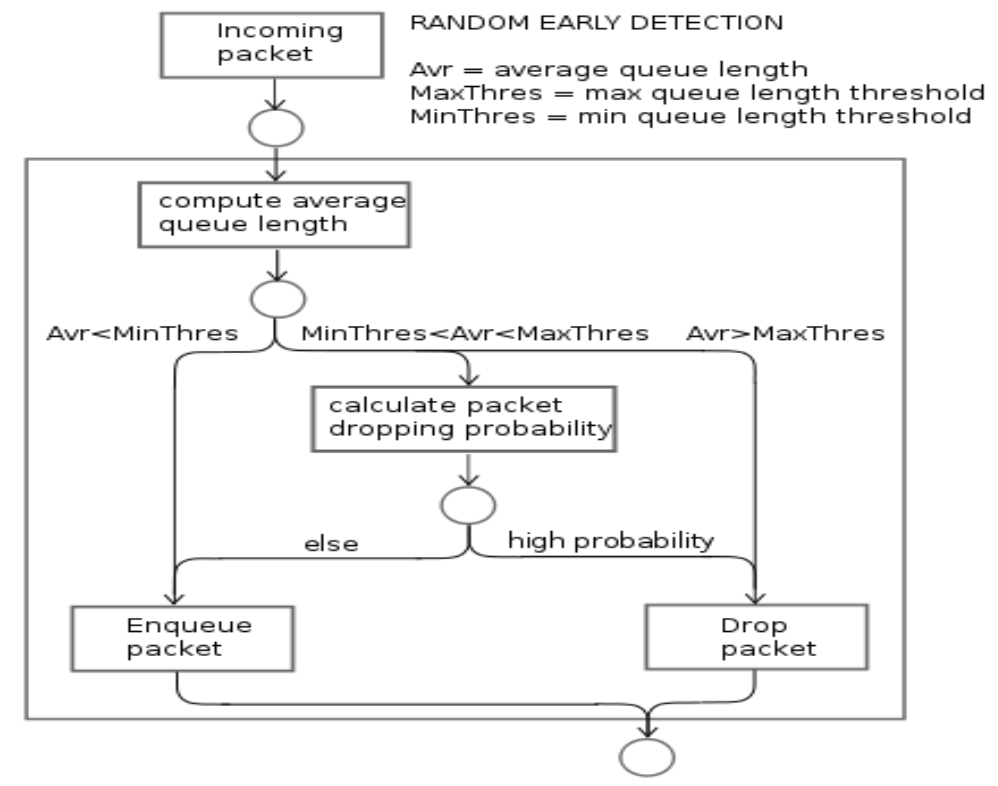

Figure 2. RED Algorithm

\section{ERED-FEC ALGORITHM}

The ERED-FEC mechanism consists of five components: (1) a packet type classifier (2) a packet loss monitor (3) a video quality model (4) a network load monitor and (5) an FEC packet generator. During video streaming, the streaming server encapsulates the video data in (RTP) Real-time Transport Protocol packets and delivers them to the receiver through the wireless AP. The ERED-FEC controller retrieves the packet header from the UDP, when the packets arrive at the AP, and identifies the packet type by checking the RTP header. Once a complete block of video packets has arrived, the packet loss monitor estimates the packet loss rate by examining the number of packet retransmissions associated with the block. The video quality model is used to determine the suitable FEC redundancy rate. Finally, the ERED FEC mechanism checks the queue length at the AP to evaluate the current network traffic load, and uses this information to adjust the FEC redundancy rate. 
1) Estimation of Packet Loss Rate in Wireless Network: In designing the ERED-FEC mechanism, it is assumed that the wireless errors are generated at a rate of $\mathrm{P}_{\mathrm{pkt}}$ in accordance with a random uniform model. In addition, an assumption is made that the MAC layer senders transmit a packet a maximum of $\mathrm{T}_{\max }$ times before discarding it. $\mathrm{P}_{\mathrm{pkt}}$ represents the failure probability of a packet transmitted just one time. However, packet retransmission increases the probability of the packet being successfully received. Therefore, the perceived probability of a packet being correctly received is given as

$\mathrm{P}_{\text {correct }}=\left(1-\mathrm{P}_{\mathrm{pkt}}\right)^{\mathrm{Tmax}}$

While, the effective failure probability of a packet is equal to

$\mathrm{P}_{\mathrm{Tmax}}=\left(\mathrm{P}_{\mathrm{pkt}}\right)^{\mathrm{Tmax}}$

In general, when the packet loss rate is high, the number of packet retransmissions is also more, and vice versa. For example, assume that a total of $\left(\mathrm{N}_{\mathrm{pkt}} \mathrm{i}\right)$ packets of the $\mathrm{i}^{\text {th }}$ video block is to be sent by the sender. Furthermore, assume that to successfully transmit all of these video packets, the total number of packet retransmissions is equal to $\left(\mathrm{RT}_{\text {total }}\right.$ ). According to the (2), with the limit of packet retransmission time $\left(\mathrm{T}_{\max }\right)$, the number of lost packets is $\left(\mathrm{N}_{\mathrm{pkt} \_} \mathrm{i} * \mathrm{P}_{\mathrm{pkt}}{ }^{\mathrm{Tmax}}\right)$. We can express the equation of the total number of correctly received packets as below:

$\left(\mathrm{N}_{\mathrm{pkt} \_\mathrm{i}}+\mathrm{RT}_{\text {total_ }} \mathrm{i}\right) *\left(1-\mathrm{P}_{\mathrm{pkt}}\right)=\mathrm{N}_{\mathrm{pkt} \_\mathrm{i}}-\mathrm{N}_{\mathrm{pkt} \_\mathrm{i}} *\left(\mathrm{P}_{\mathrm{pkt}}\right)^{\mathrm{T} m a x}$

2) Analytical Model for Estimating Effective Packet Loss Rate with FEC Recovery:

Consider a video streaming file containing a total of video packets. Assuming that each transmission block comprises video packets, the video file is transmitted in a total of approximately blocks. Given an actual packet loss rate of $\mathrm{P}_{\mathrm{pkt}}$, the probability of a block being successfully recovered is given by

$$
\left(1-\mathrm{P}_{\text {block }}\right)=\sum_{k=0}^{k+h} \mathrm{C}_{\mathrm{i}}^{\mathrm{k}+\mathrm{h}} *\left(1-\mathrm{P}_{\mathrm{pkt}}\right)^{\mathrm{i}} *\left(\mathrm{P}_{\mathrm{pkt}}\right)^{\mathrm{k}+\mathrm{h}-\mathrm{i}}
$$

Where $\mathrm{P}_{\text {block }}$ is the probability of the block being unrecovered and $\mathrm{C}_{\mathrm{i}}^{\mathrm{k}+\mathrm{h}}$ denotes all possible combinations of $\mathrm{i}$ packets successfully received in a whole block. In other words, the probability that a block cannot be recovered is given by

$\mathrm{P}_{\text {block }}=1-\sum_{k=0}^{k+h} \quad \mathrm{C}_{\mathrm{i}}^{\mathrm{k}+\mathrm{h}} *\left(1-\mathrm{P}_{\mathrm{pkt}}\right)^{\mathrm{i}} *\left(\mathrm{P}_{\mathrm{pkt}}\right)^{\mathrm{k}+\mathrm{h}-\mathrm{i}}$

Since the video file comprises approximately blocks, the expected number of successfully recovered blocks can be estimated as

$\frac{\boldsymbol{N}}{\boldsymbol{k}} *\left(1-\mathrm{P}_{\text {block }}\right)=\frac{\boldsymbol{N}}{\boldsymbol{k}} * \sum_{\boldsymbol{k}=0}^{\boldsymbol{k}+\boldsymbol{h}} \quad \mathrm{C}_{\mathrm{i}}^{\mathrm{k}+\mathrm{h}} *\left(1-\mathrm{P}_{\mathrm{pkt}}\right)^{\mathrm{i}} *\left(\mathrm{P}_{\mathrm{pkt}}\right)^{\mathrm{k}+\mathrm{h}-\mathrm{I}}$

While the expected number of unrecoverable blocks is given by

$\frac{\boldsymbol{N}}{\boldsymbol{k}} * \mathrm{P}_{\text {block }}=\frac{\boldsymbol{N}}{\boldsymbol{k}} *\left[1-\sum_{\boldsymbol{k}=0}^{\boldsymbol{k}+\boldsymbol{h}} \mathrm{C}_{\mathrm{i}}^{\mathrm{k}+\mathrm{h}} *\left(1-\mathrm{P}_{\mathrm{pkt}}\right)^{\mathrm{i}} *\left(\mathrm{P}_{\mathrm{pkt}}\right)^{\mathrm{k}+\mathrm{h}-\mathrm{i}}\right]$

From (6) and (7), the expected number of packets received by the receiver application can be estimated as

$\frac{\boldsymbol{N}}{\boldsymbol{k}} *\left(1-\mathrm{P}_{\text {block }}\right) * \mathrm{k}+\frac{\boldsymbol{N}}{\boldsymbol{k}} * \mathrm{P}_{\text {block }} *(\mathrm{k}-[(\mathrm{h}+1) * \mathrm{k} / \mathrm{k}+\mathrm{h}])$

The first and second terms in (8) give the total number of video packets received from the successfully recovered blocks and the unrecovered blocks, respectively.

\section{SIMULATION RESULT}

In order to create our Simulation model, we used NS2 version 2.35 simulation environment with the installation of the all-inone package that operate on Ubuntu through VMware server (16). We consider several scenarios and evaluated the performance of the proposed ERED-FEC mechanisms, which are implemented with scripts written in TCL that comprise commands and parameters for simulator initialization, node creation and configuration. 
Table -1 . The NS2 simulation environment

\begin{tabular}{|l|l|}
\hline Simulator & Network Simulator \\
\hline Simulation of nodes & 50 \\
\hline Interface type & Phy/wirelessPhy \\
\hline Channel & Wireless channel \\
\hline Mac type & MAC_802.11 \\
\hline Queue type & Queue/PrioQueue/Droptail \\
\hline Queue length & 60 Packets \\
\hline Retrylimit(Tmax) & 4 \\
\hline Queue management policy & RED \\
\hline Antenna type & OmniAntenna \\
\hline Propogation model & TwoRayGround \\
\hline Size of packet & 512 \\
\hline Protocol & AODV \\
\hline Traffic & TCP \\
\hline
\end{tabular}

\subsection{Throughput}

It is defined as the total amount of data received by destination from the source node divided by the time taken to receive the last packet. Figure 3 shows the graph of Time (X-Axis) versus Throughput (Y-Axis). It has been clear from the figure that the proposed algorithm gives higher throughput than the Traditional mechanisms. Once the average queue size crosses the minimum threshold value, packet drop takes place in order to avoid congestion hence in that case throughput gradually flows down and once the packet drop remains constant even the throughput remains constant.

\subsection{Packet Delivery Ratio}

It is the total number of packets received by Destination to total number of packets sent by the Source. Figure 4 shows the graph of time (X-Axis) versus packet delivery ratio (Y-Axis). The graph shows that initially PDR is more as there is no packet drop. When the Avg crosses the minimum threshold value, packet drop increases gradually for getting away from congestion; in that case PDR gradually decreases. When the packet dropping reduces, there after slightly PDR increases and when the packet drop remains constant then PDR also remains constant.

Fig.4. Show the variation of the AP queue length and the number of generating FEC redundant packets under light to heavy network load condition. Note that the packet loss rate is specified as 0.5 and the FEC rate $(\mathrm{n}, \mathrm{k})$ is set as $(15,8)$. Under a light to heavy load, the mean queue length is 12.8 packets and the mean number of FEC redundant packets per block is equal to 4.66.

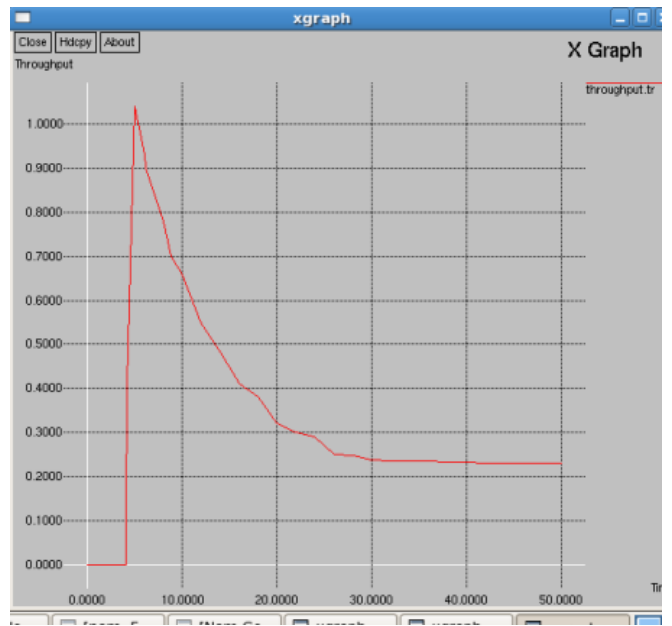

Figure 3. Throughput

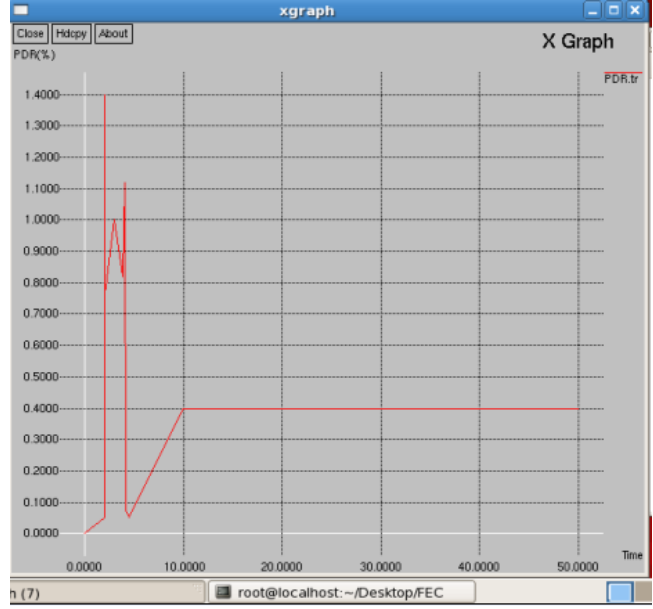

Figure 4. Packet Delivery Ratio

\section{CONCLUSION}

This paper has presented the AP based packet level FEC mechanism for improving the data transmission with the use of RED algorithm. In the proposed approach, the RED-FEC algorithm tunes the number of redundant FEC packets in accordance with the access point queue length in such a way that the redundant packets injected for loss recovery purposes do not result in network congestion. Hence we get the improved performance like throughput, packet delivery ratio. The recovery performance of the ERED-FEC mechanism will be further enhanced by utilizing a hybrid RED algorithm over Wi-Max, WLAN etc. 


\section{REFERENCES}

[1] Cheng-Han Lin, Ce-Kuen Shieh, Wen-Shyang Hwang, "An Access Point-Based FEC Mechanism for Video Transmission Over Wireless LANs", IEEE Trans. Multimedia, Vol. 15, pp. 195-206, Jan 2013.

[2] H. Zhihai, X. Hongkai,"Transmission distortion analysis for real-time video encoding and streaming over wireless networks,"IEEE Trans. Circuits Syst. Video Technol., Vol. 16, pp. 1051-1062, 2006.

[3] A. Nafaa, Y. Hadjadj-Aoul, A. Mehaoua,"On interaction Between loss characterization and forward error correction in wireless multimedia communication", In Proc. IEEE Int.

[4] E. Maani and A. Katsaggelos, "Unequal error protection for robust streaming of scalable video over packet lossy networks," IEEE Trans. Circuits Syst. Video Technol., vol. 20, pp. 407-416, 2010.

[5] A. Argyriou, "Cross-layer error control for multimedia streaming in wireless/wireline packet networks FEC scheme for TDM-OFDM based satellite radio broadcasting syste m,” IEEE Trans. Multimedia, vol. 10, pp. 1121-1127, Oct. 2008.

[6] J. Dan, F. Pascal, and J. Aleksandar, "Forward error correction for multipath Media streaming," IEEE Trans. Circuits Syst. Video Technol., vol. 19, pp. 1315-1326, 2009.

[7] J. Paavola, H. Himmanen, T. Joela, J. Poikonen, and V. Ipatov, "The performance analysis of MPE-FEC decoding methods at the DVB-H link layer for efficient IP packet retrieval,” IEEE Trans. Broadcast., vol. 53, pp. 263-275, Mar. 2007.

[8] J. Paavola, H. Himmanen, T. Joela, J. Poikonen, and V. Ipatov, "The performance analysis of MPE-FEC decoding methods at the DVB-H link layer for efficient IP packet retrieval,” IEEE Trans. Broadcast., vol. 53, pp. 263-275, Mar. 2007.

[9] M. van der Schaar and D. S. Turaga, "Cross-Layer Packetization and Retransmission Strategies for Delay-Sensitive Wireless Multimedia Transmission," Multimedia, IEEE Transactions on, vol. 9, no. 1, pp. 185 -197, Jan. 2007.

[10] A. Nafaa, T. Ahmed, and A. Mehaoua, "Unequal and interleaved FEC protocol for robust MPEG-4 multicasting over wireless LANs," in Proc. IEEE Conf. Computer and Communications, ICC2004, Jun. 2004, vol. 3, pp. 1431-1435.

[11] A. Nafaa, T. Taleb, and L. Murphy, "Forward error correction strategies for media streaming over wireless networks," Communications Magazine, IEEE, vol. 46, no. 1, pp. $72-79$, Jan. 2008.

[12] A. Basalamah and T. Sato, "A Comparison of Packet-Level and Byte-Level Reliable FEC Multicast Protocols for WLANs," in IEEE Global Telecommunications Conference, 2007. GLOBECOM '07, 2007, pp. $4702-4707$.

[13] Z. Li, A. Khisti, and B. Girod, "Forward Error Protection for low-delay packet video," in Packet Video Workshop (PV), 2010 18th International, 2010, pp. $1-8$.

[14] D. Wu, Y. T. Hou, and Y.-Q. Zhang, "Transporting real-time video over the Internet: challenges and approaches," Proceedings of the IEEE, vol. 88, no. 12, pp. $1855-1877$, Dec. 2000.

[15] S. Floyd and V. Jacobson, "Random early detection gateways for congestion avoidance," IEEE/ACM Trans. Networking, vol. 4, pp.397-413, Aug. 1993.

[16] “NS-2 Simulator,” [Online]. Available: http://hpds.ee.ncku.edu.tw/smallko/ns2/ns2.html. 